\title{
High-Sensitivity Temperature Sensor Based on Polarization Maintaining Fiber Sagnac Loop
}

\author{
Laipeng $\mathrm{SHAO}^{1}$, Junhui $\mathrm{HU}^{2}$, Hanglin $\mathrm{LU}^{2}$, Jing $\mathrm{DU}^{2}$, \\ Tianyin $\mathrm{WU}^{2}$, and Yiping $\mathrm{WANG}^{1^{*}}$
}

\author{
${ }^{1}$ Key Laboratory of Optoelectronic Devices and System of Ministry of Education and Guangdong Province, College of \\ Optoelectronic Engineering, Shenzhen University, Shenzhen 518060, China \\ ${ }^{2}$ College of Physics Science and Technology, Guangxi Normal University, Guilin 541001, China \\ *Corresponding author: Yiping WANGＩ-mail: ypwang@szu.edu.cn
}

\begin{abstract}
A high-sensitivity all-fiber temperature sensor based on a Sagnac interferometer is demonstrated by splicing a section of polarization maintaining fiber (PMF) between two sections of standard single mode fibers (SMFs). In this sensor, the SMF-PMF-SMF structure in the Sagnac loop is bent into a circle to enhance the sensitivity. The length and curvature of the PMF in the loop are investigated and can be optimized to further increase the temperature sensitivity of the sensor. Results show that the radius of the circle has an important effect upon temperature sensitivity due to the bend-induced birefringence variation of the PMF. The SMF-PMF-SMF structure bent into a circle with a radius of $30 \mathrm{~mm}$ exhibits a high-sensitivity temperature of $1.73 \mathrm{~nm} /{ }^{\circ} \mathrm{C}$. The sensor is provided with the advantages of easy fabrication, low-insertion loss, and high sensitivity, which may find potential applications in the field of high precision temperature measurement.
\end{abstract}

Keywords: Optical fiber sensor; polarization maintaining fiber; temperature sensor; Sagnac loop

Citation: Laipeng SHAO, Junhui HU, Hanglin LU, Jing DU, Tianyin WU, and Yiping WANG, "High-Sensitivity Temperature Sensor Based on Polarization Maintaining Fiber Sagnac Loop," Photonic Sensors, 2019, 9(1): 25-32.

\section{Introduction}

Interferometric fiber optic sensors combined with mode interference structure and Sagnac loop have shown the advantages of high sensitivity, stable and simple structure, and compatibility and low prices [1-3]. This type of interferometer can be fabricated by splicing a segment of multimode fiber (MMF) between two standard single mode fibers (SMFs), i.e. the SMF-MMF-SMF structure. The transmission principle of the SMS structure as to the mode of MMF is excited by the external perturbation to induce multimode interference, from which the external environment parameter of change can be measured. Due to the MMF with unique transmission advantages, Gong et al. [4] proposed an all-fiber curvature sensor exhibiting high sensitivity that can be obtained over a relatively large scale of curvature. Apparently, the development based on the SMS structure has been extensively studied for measuring strain [5], displacement [6], refractive index [7], and magnetic field [8]. Many efforts have been made to enhance the temperature sensitivity of temperature sensors based on the SMS structure. Liu et al. [9] proposed a high-sensitivity temperature and strain sensor based on mode interference in graded-index multimode fibers, and a temperature sensitivity of $58.5 \mathrm{pm} /{ }^{\circ} \mathrm{C}$

Received: 5 September 2018 / Revised: 14 September 2018

(C) The Author(s) 2018. This article is published with open access at Springerlink.com

DOI: $10.1007 / \mathrm{s} 13320-018-0517-6$

Article type: Regular 
was obtained in their structure. Wu et al. [10] showed a high temperature sensitivity of $32 \mathrm{pm} /{ }^{\circ} \mathrm{C}$ based on a bent SMS structure employed. Zhang et al. [11] proposed and experimentally investigated a temperature sensor based on a bent SMS structure. The sensor showed a high sensitivity of $6.5 \mathrm{~nm} /{ }^{\circ} \mathrm{C}$. However, the cladding of MMF was etched by a chemical method that it was substituted with a liquid material. Consequently, the fabrication of sensor was relatively complex. Lim et al. [12] reported that a microfiber device integrating a microfiber knot resonator in a Sagnac loop reflector would obtain a temperature sensitivity of $20.6 \mathrm{pm} /{ }^{\circ} \mathrm{C}$. Shi et al. [13] demonstrated that a temperature sensor based on a fiber ring laser with a reflective Sagnac loop could obtain a sensitivity of $1.739 \mathrm{~nm} /{ }^{\circ} \mathrm{C}$. Reyes et al. [14] showed a high-sensitivity temperature sensor based on an all-fiber Sagnac loop interferometer. The temperature sensor with metal-filled side-hole photonic crystal fiber could reach up to the sensitivity of $-9 \mathrm{~nm} /{ }^{\circ} \mathrm{C}$. Hence, the expensive photonic crystal fiber was used, and the filling operation was complicated in their scheme. More recently, Ruan et al. [15] proposed a temperature sensor based on a circled SMPMS-fiberstructure-based Saganc loop. Although the sensor showed a temperature sensitivity of $1.476 \mathrm{~nm} /{ }^{\circ} \mathrm{C}$, this structure had four splicing points which led to a great loss in insertion.

In this paper, a high-sensitivity temperature sensor based on the Sagnac interferometer is demonstrated by splicing a section of polarization maintaining fiber (PMF) between two standard SMFs. Such an SMF-PMF-SMF structure is bent into a circle of different radii in the Saganc loop. The effects of the length and bent curvature with the PMF employed on the sensing properties are investigated in detail to enhance the temperature sensitivity of the PMF-based Sagnac loop interferometer. Our proposed sensor exhibits a high temperature sensitivity of $1.73 \mathrm{~nm} /{ }^{\circ} \mathrm{C}$ when the length and bent curvature with the PMF employed are $150 \mathrm{~mm}$ and $30 \mathrm{~mm}$, respectively. Such a high-sensitivity temperature sensor based on a
Saganc loop with a bent PMF could find some applications in the health monitor of large engineering structures.

\section{Experimental setup and principle}

The schematic of PMF-Sagnac interferometer temperature sensor is shown in Fig. 1. The fiber-optic temperature sensor is fabricated with a segment of PMF (PMF1550-HP) between standard SMFs (G652D) and is structured with a Sagnac loop. A broadband light source (BBS) ranging from $1310 \mathrm{~nm}$ to $1650 \mathrm{~nm}$ is used as a light source in the experiments. The light emitted from the BBS is divided into two counter-propagating light beams by a 3-dB coupler. These two light beams propagate through the SMF-PMF-SMF structure and generate a phase difference due to the birefringence of the PMF. The interference spectrum can be formed when the two light beams are recombined at the output port of the $3-\mathrm{dB}$ coupler and then is measured by an optical spectrum analyzer (OSA, Yokagawa, AQ6370C).

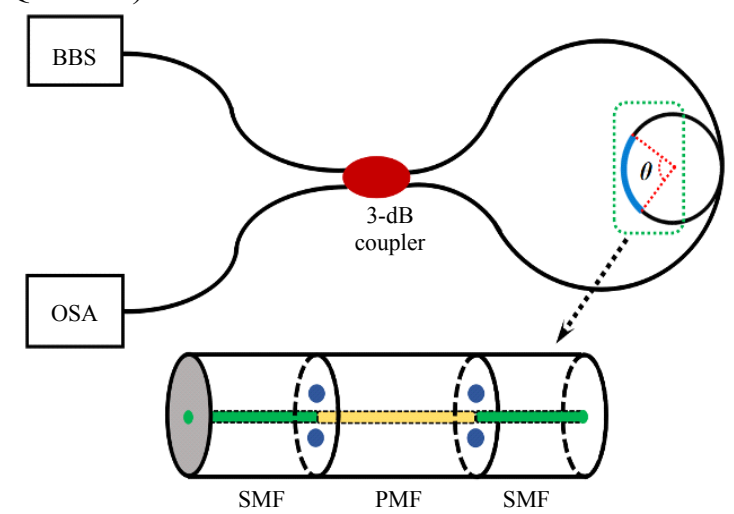

Fig. 1 Schematic diagram of Saganc interferometer based on a polarization maintaining fiber with a circle.

The PMF used in our experiments is characterized by a germanium-doped core and two boron-doped circular regions symmetrically located on both sides of the core, and the beat length at $1550 \mathrm{~nm}$ is $3 \mathrm{~mm}$. The SMF-PMF-SMF structure is similar to that described in [15].

Ignoring the loss of the Sagnac loop, the transmission optical intensity $I$ in terms of the phase difference can be described as [16]

$$
I=\frac{I_{\text {in }}(1-\cos \varphi)}{2}
$$


and

$$
\varphi=\frac{2 \pi B(R, T) L}{\lambda}
$$

where $\varphi$ is the total phase difference produced by the two beams in the return coupler, $\lambda$ and $I_{\text {in }}$ are the wavelength and intensity of incident light, respectively, $L$ is the length of the PMF, and $B(R, T)$ is the birefringence induced by PMF bending and temperature raising. The transmission dip wavelength is the resonant wavelength satisfying $2 \pi B(R, T) L / \lambda=2 k \pi$, and $k$ is a random integer. The temperature-induced and the bend-induced birefringence change in the PMF resulting in the wavelength shift sensitivity respectively can be expressed as follows:

$$
\begin{gathered}
\frac{d \lambda_{T}}{d T}=\frac{d B(R, T)}{d T} \frac{\lambda}{B(R, T)}+\frac{d L}{d T} \frac{\lambda}{L} \\
\frac{d \lambda_{R}}{R}=\frac{d B(R, T)}{d R} \frac{\lambda}{B(R, T)} .
\end{gathered}
$$

The bend-induced birefringence change is given by [17]

$$
\frac{d B(R, T)}{d R}=0.5 C_{s}\left(\frac{r}{R}\right)^{2}
$$

where

$$
C_{s}=0.5 k_{0} n_{0}^{3}\left(p_{11}-p_{12}\right)(1+v)
$$

in which $r$ and $R$ are the radii of the PMF and bend, respectively, $C_{s}$ is a parameter combination of PMF, and $v$ is Poisson's ratio. The amount of the length $L$ change in PMF caused by the temperature variation can be negligible due to the small expansion coefficient of quartz. From (3) and (4), when the bending is applied to the sensor and the temperature rises, it would cause the resonant dip wavelength shift $\Delta \lambda_{\text {dip }}$, which can be written as follows:

$$
\begin{aligned}
\Delta \lambda_{\text {dip }} & =\frac{d \lambda_{T}}{d T}+\frac{d \lambda_{R}}{R} \\
& =\frac{d B(R, T)}{d T} \frac{\lambda}{B(R, T)}+\frac{d B(R, T)}{d R} \frac{\lambda}{B(R, T)} \\
& =\frac{\lambda}{B(R, T)}\left[\frac{d B(R, T)}{d T}+0.5 C_{s}\left(\frac{r}{R}\right)^{2}\right] .
\end{aligned}
$$

Equation (7) shows that the dip wavelength shift is influenced by two terms: intrinsic temperatureinduced birefringence change which is related to the nature of the fiber and the ratio between the PMF radius and the bend radius. The influence of the length is implicit in (7) and should play a role too.

\section{Experimental results and discussion}

In order to optimize the length of PMF, we first investigate the temperature sensitivity dependence of the PMF length before the SMF-PMF-SMF structure is bent into a circle. At room temperature, we observe that the characteristic transmission spectra of PMFs have the lengths of $120 \mathrm{~mm}$, $150 \mathrm{~mm}, 170 \mathrm{~mm}$, and $200 \mathrm{~mm}$ as shown in Fig. 2. In the experiments, the SMF-PMF-SMF structurebased Sagnac loop is placed in an incubator (WD2005) with a resolution of $0.1{ }^{\circ} \mathrm{C}$. The transmission spectrum change of the sensor has been responded to the temperature range from $30{ }^{\circ} \mathrm{C}$ to $42{ }^{\circ} \mathrm{C}$ with a step of $2{ }^{\circ} \mathrm{C}$ and is measured by an OSA with the resolution of $0.1 \mathrm{~nm}$ after 5 minutes for thermal equilibrium. We choose the resonant dip wavelength near the $1550 \mathrm{~nm}$ of the sensor for measurement.

The applied temperature is increased from $30^{\circ} \mathrm{C}$ to $42{ }^{\circ} \mathrm{C}$ with the temperature interval of $2{ }^{\circ} \mathrm{C}$. As shown in Fig. 3, PMF-based Sagnac interferometer with different PMF lengths has the typical transmission spectra blue-shift with the change of temperature. The plots of the dips wavelength shift in response to the temperature change are shown in Fig. 4(a). The employed Saganc interferometer has different PMF lengths: $120 \mathrm{~mm}, 150 \mathrm{~mm}, 170 \mathrm{~mm}$, and $200 \mathrm{~mm}$, respectively. The results show that the temperature sensitivities of all sensors are little with difference. Therefore, from Fig. 4(b), the PMF length of $150 \mathrm{~mm}$ is the optimized PMF length, which gives the best temperature sensitivity of $1.53 \mathrm{~nm} /{ }^{\circ} \mathrm{C}$. 


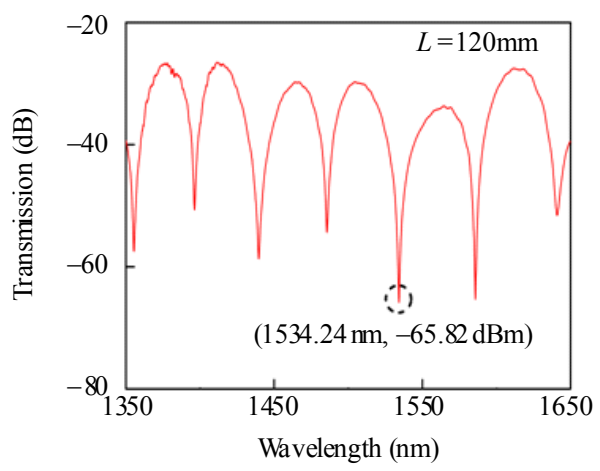

(a)

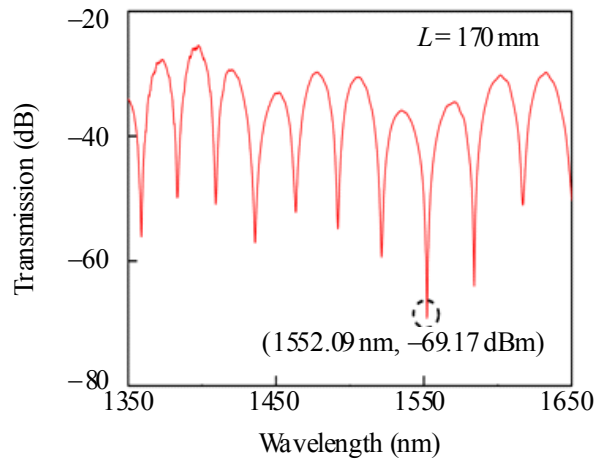

(c)

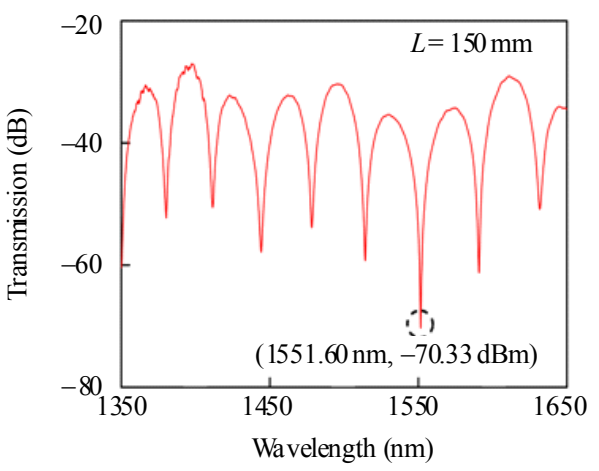

(b)

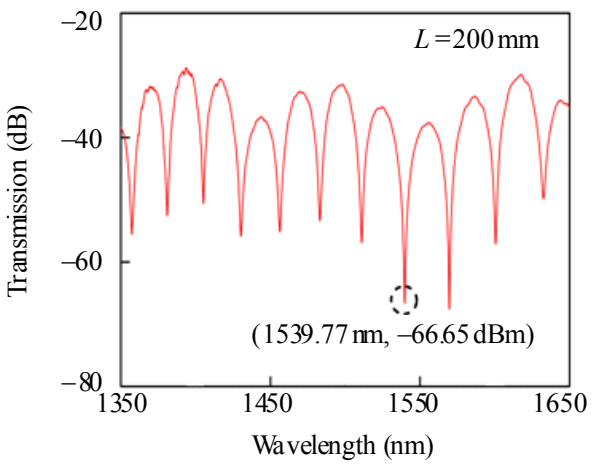

(d)

Fig. 2 Transmission spectra of the PMF-based Saganc interferometer with different PMF lengths of (a) $120 \mathrm{~mm}$, (b) $150 \mathrm{~mm}$, (c) $170 \mathrm{~mm}$, and (d) $200 \mathrm{~mm}$ at room temperature.

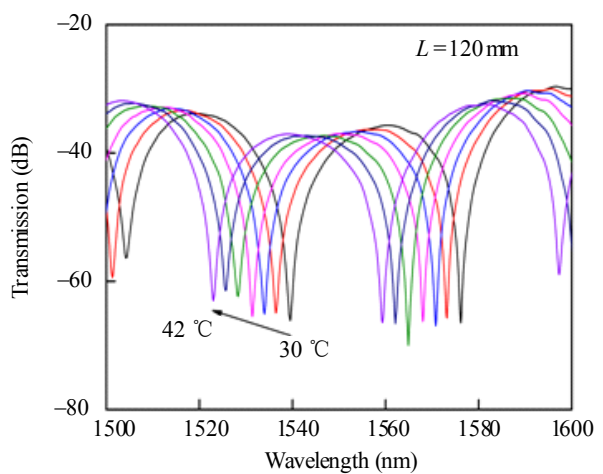

(a)

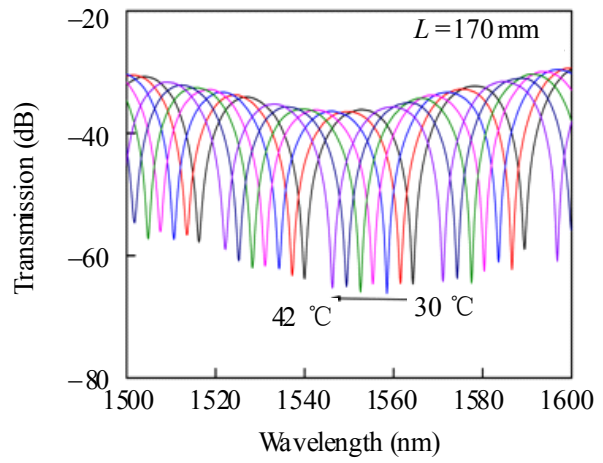

(c)

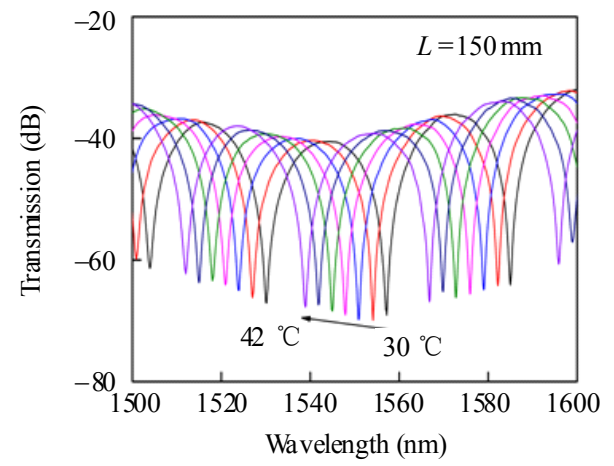

(b)

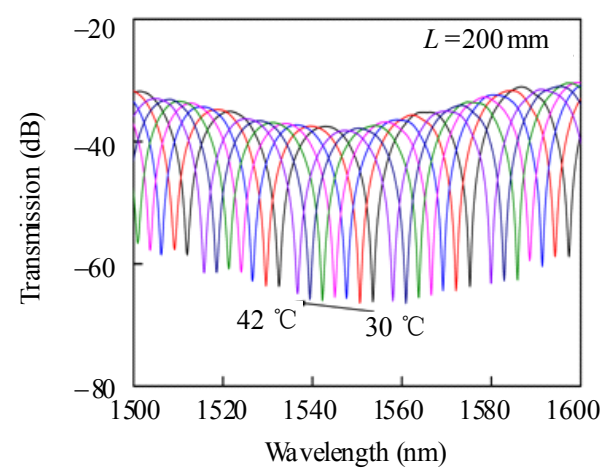

(d)

Fig. 3 Transmitted spectra shift of the PMF-based Sagnac interferometer with different PMF lengths of (a) $120 \mathrm{~mm}$, (b) $150 \mathrm{~mm}$, (c) $170 \mathrm{~mm}$, and (d) $200 \mathrm{~mm}$ while the temperature rises from $30{ }^{\circ} \mathrm{C}$ to $42{ }^{\circ} \mathrm{C}$. 


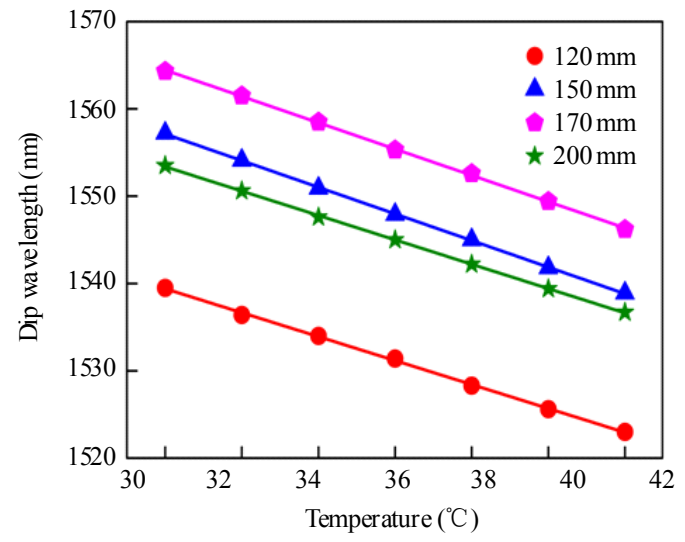

(a)

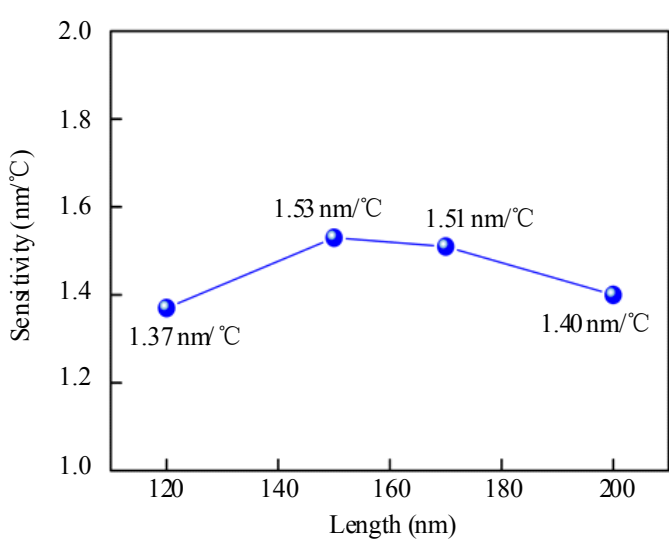

(b)

Fig. 4 PMF-based Sagnac interferometer has (a) the dependence on temperature of different PMF lengths and (b) the different distribution of temperature sensitivity.

We bent the SMF-PMF-SMF structure with different radii of a circle in the Saganc loop in the following experiments. For the purpose of enhancing the temperature sensor sensitivity, the SMF-PMF-SMF structure with $150 \mathrm{~mm}$ length of PMF is bent into a circle employed. In the experiments, the SMF-PMF-SMF structure is successively bent into a circle with the radii of $30 \mathrm{~mm}, 50 \mathrm{~mm}, 70 \mathrm{~mm}, 100 \mathrm{~mm}, 120 \mathrm{~mm}$, and $150 \mathrm{~mm}$, then fixed in an incubator.

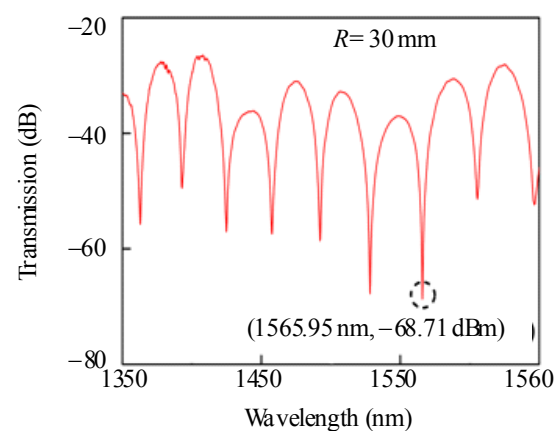

(a)

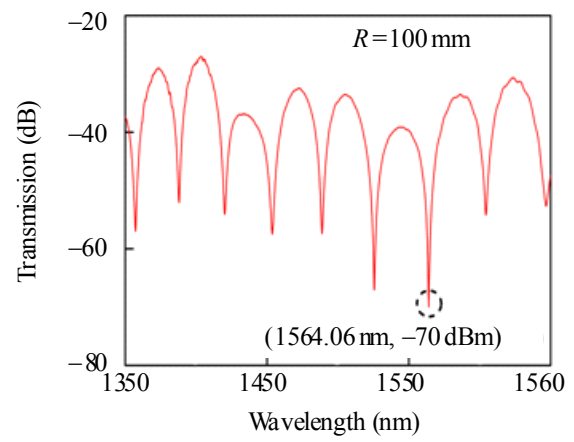

(d)

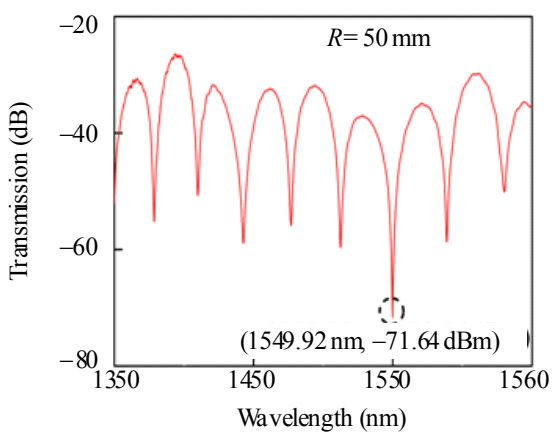

(b)

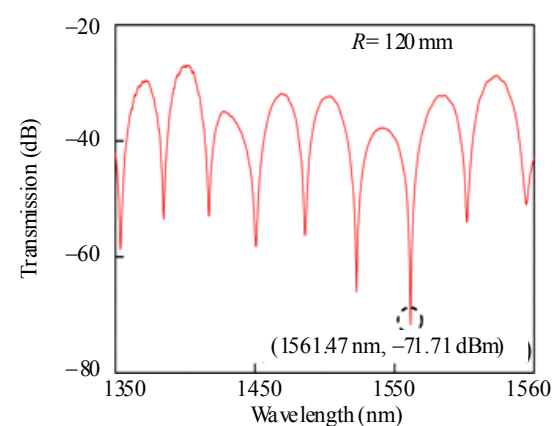

(e)
From Fig. 5, we can see transmission spectra of the sensor under different radii of circle at room temperature. Similarly, we select the resonant dip wavelength near $1550 \mathrm{~nm}$ as the observation when the temperature ranges from $30{ }^{\circ} \mathrm{C}$ to $50{ }^{\circ} \mathrm{C}$ with temperature intervals of $5{ }^{\circ} \mathrm{C}$. At each temperature increment, we acquire the respective transmission spectra by using an optical spectrum analyzer (OSA) after 5 minutes for thermal equilibrium.

Fig. 5 Transmitted spectra of the PMF-based Sagnac interferometer with different PMF circle radii of (a) $30 \mathrm{~mm}$, (b) $50 \mathrm{~mm}$, (c) $70 \mathrm{~mm}$, (d) $100 \mathrm{~mm}$, (e) $120 \mathrm{~mm}$, and (f) $150 \mathrm{~mm}$ at room temperature. 


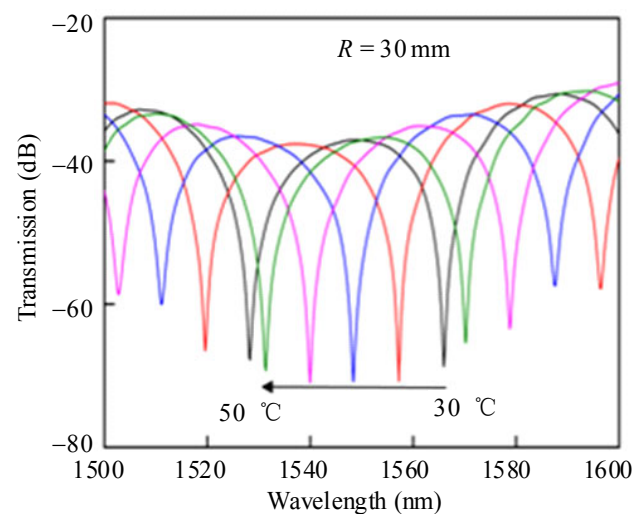

(a)

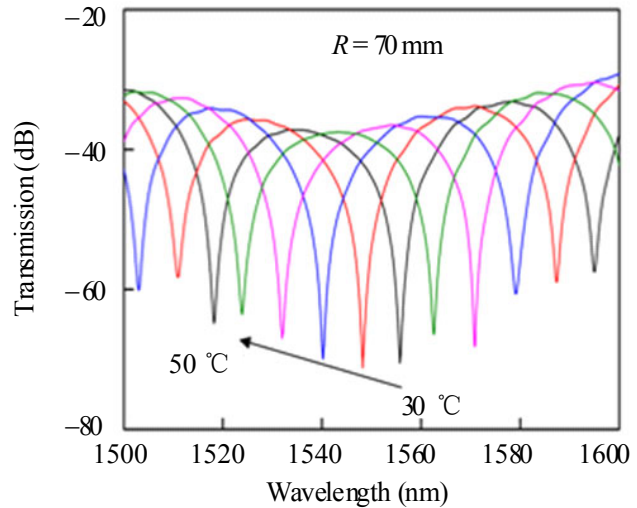

(c)

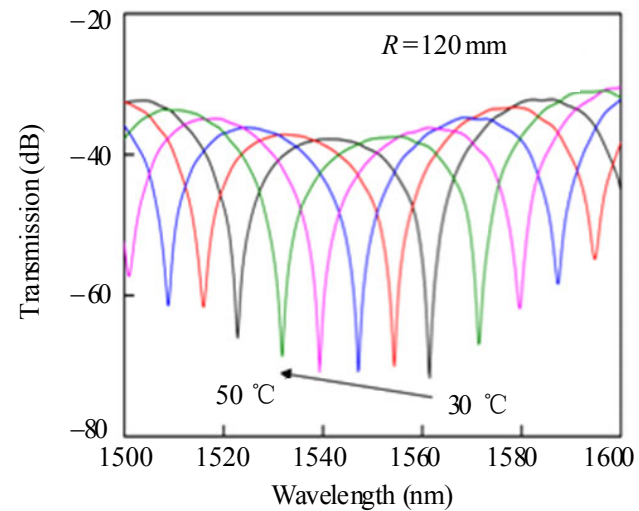

(e)

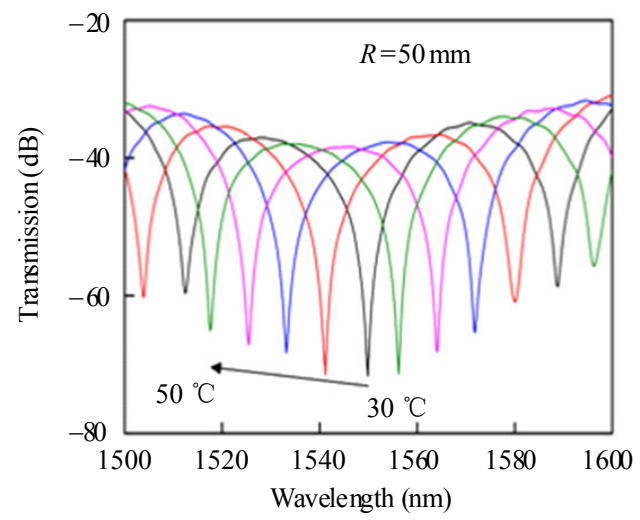

(b)

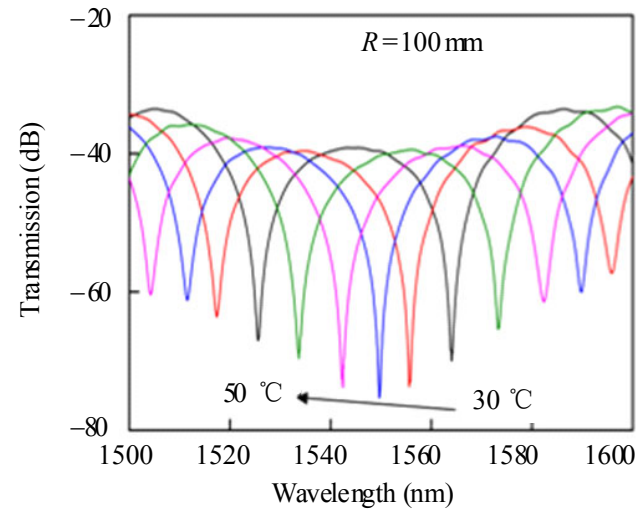

(d)

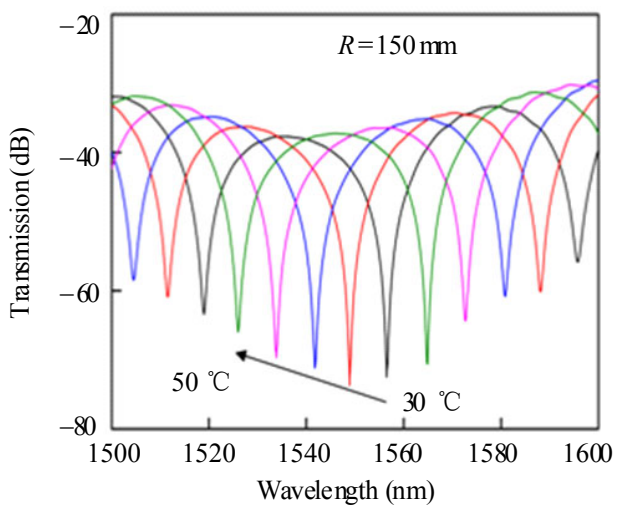

(f)

Fig. 6 Transmitted spectrum shift of the PMF-based Sagnac interferometer with different PMF circle radii of (a) $30 \mathrm{~mm}$, (b) $50 \mathrm{~mm}$, (c) $70 \mathrm{~mm}$, (d) $100 \mathrm{~mm}$, (e) $120 \mathrm{~mm}$, and (f) $150 \mathrm{~mm}$ at room temperature.

It is obvious to see that, the spectra shifts of the sensor have the response to the temperature rises for different radii as shown in Fig. 6. The transmission spectra for different circle radii show blue-shift characteristics while the temperature rises.

As shown in Fig. 7(a), we plot the fringe dip wavelength of the PMF-based Saganc interferometer under different PMF circle radii at different temperatures and the dip wavelength shifts have a linear response when the temperature rises. When the radii of the PMF circle are $30 \mathrm{~mm}, 50 \mathrm{~mm}$, $70 \mathrm{~mm}, 100 \mathrm{~mm}, 120 \mathrm{~mm}$, and $150 \mathrm{~mm}$, the temperature sensitivities of PMF-based Saganc interferometer are $1.73 \mathrm{~nm} /{ }^{\circ} \mathrm{C}, \quad 1.61 \mathrm{~nm} /{ }^{\circ} \mathrm{C}$, $1.60 \mathrm{~nm} /{ }^{\circ} \mathrm{C}, 1.48 \mathrm{~nm} /{ }^{\circ} \mathrm{C}, 1.49 \mathrm{~nm} /{ }^{\circ} \mathrm{C}$ and $1.52 \mathrm{~nm} /{ }^{\circ} \mathrm{C}$, respectively. Obviously, the maximum sensitivity of $1.73 \mathrm{~nm} /{ }^{\circ} \mathrm{C}$ is obtained when the circle radius is $30 \mathrm{~mm}$, which is higher than that in [15] due to the 
smaller radius of the reeled circle is employed in our experiment. As shown in Fig. 7(b), it is obvious that the temperature sensitivity is approximately inversely proportional to the curvature radius $R$, which better agrees with the theoretical analysis in (7). It is found from Figs. 4(b) and 7(b) that the temperature sensitivity of the sensor increases from $1.53 \mathrm{~nm} /{ }^{\circ} \mathrm{C}$ to $1.73 \mathrm{~nm} /{ }^{\circ} \mathrm{C}$ after the PMF with a length of $150 \mathrm{~mm}$ is bent. Our experiments show that the bend-induced loss increases rapidly when the bent curvature of the PMF decreases to $20 \mathrm{~mm}$. So the optimum bent curvature of the PMF is $30 \mathrm{~mm}$ for a desired sensor with a high temperature sensitivity of $1.73 \mathrm{~nm} /{ }^{\circ} \mathrm{C}$ and a low insertion loss of less than $1.0 \mathrm{~dB}$. Therefore, our proposed high-sensitivity temperature sensor based on a Saganc loop with a bent PMF could find some applications in the health monitor of large engineering structures.

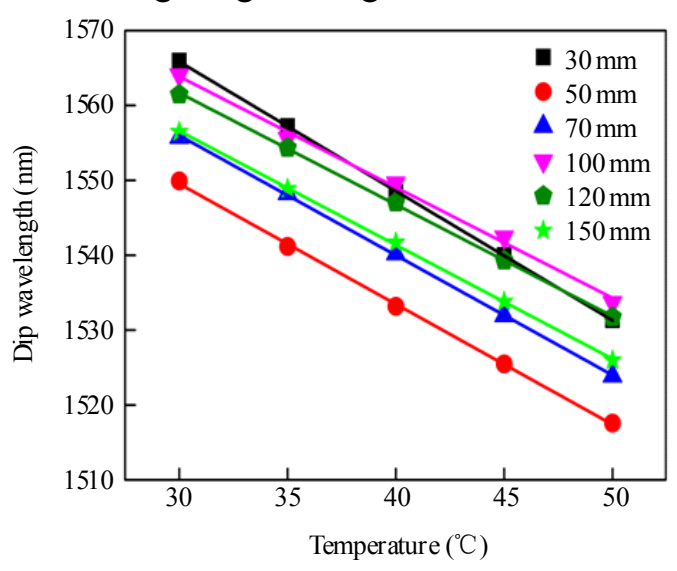

(a)

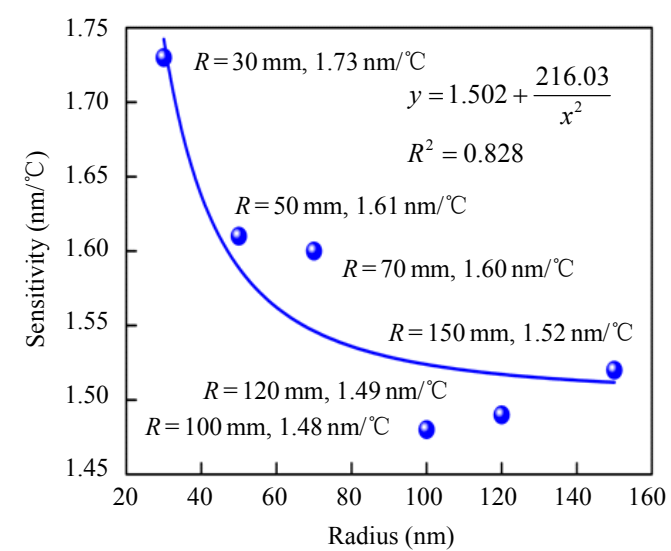

(b)

Fig. 7 PMF based-Sagnac interferometer of the PMF length is $150 \mathrm{~mm}$ with (a) the different circle radii dependence on temperature response (b) and the different temperature sensitivity distribution on different circle radii.
The temperature sensitivity of the proposed PMF-based Saganc loop interferometer is in general dependent on the state of polarization (SOP) of the two lights due to the phase shift [18], which has been indicated by the experimental results illustrated in Figs. 4(b) and 7(b). We will experimentally investigate in detail the effect of the SOP of the two lights on the temperature sensitivity of the proposed sensor configuration in the future.

\section{Conclusions}

We have proposed and demonstrated a high-sensitivity all-fiber temperature sensor based on the circled SMF-PMF-SMF embedded Saganc loop. The temperature sensitivity dependence on the radius of the reeled circle has been experimentally and theoretically investigated. The sensor exhibits a high temperature sensitivity of $1.73 \mathrm{~nm} /{ }^{\circ} \mathrm{C}$ when the bending radius of the circle is $30 \mathrm{~mm}$, which is much higher than that of previously reported fiber sensors. Moreover, this sensor shows advantages of easy fabrication and low insertion loss, which could find potential applications in temperature measurements.

\section{Acknowledgement}

This work was supported by the National Natural Science Foundation of China (NSFC) (Grant Nos. 61425007 and 61635007); Guangdong Natural Science Foundation (Grant Nos. 2015B010105007 and 2014A030308007); Science and Technology Innovation Commission of Shenzhen (Grant Nos. JCYJ20170412105604705 and JCYJ20160427104925452); Development and Reform Commission of Shenzhen Municipality Foundation; the National Natural Science Foundation of China (Grant No. 61565002); Guangxi Province Key Research and Development Program (Grant No. AB171129027).

Open Access This article is distributed under the terms of the Creative Commons Attribution 4.0 International License (http://creativecommons.org/licenses/by/4.0/), which permits unrestricted use, distribution, and reproduction in any medium, provided you give 
appropriate credit to the original author(s) and the source, provide a link to the Creative Commons license, and indicate if changes were made.

\section{References}

[1] X. Wang, H. J. Yang, S. S. Wang, Y. P. Liao, and Y. Zhang, "Seawater temperature measurement based on a high-birefringence elliptic fiber Saganc loop," IEEE Photonic Technology Letters, 2015, 27(16): 1772-1175.

[2] Y. Zhao, X. Liu, R. Q. Lv, and Q. Wang, "Simultaneous measurement of RI and temperature based on the combination of Sagnac loop mirror and balloon-like interferometer," Sensors and Actuators B, 2017, 243: 800-805.

[3] L. Y. Shao, X. P. Zhang, H. J. He, Z. Y. Zhang, X. H. Zou, B. Luo, et al., "Optical fiber temperature and torsion sensor based on Lyot-Saganc interferometer," Sensors, 2016, 16(10): 1774-1-1774-7.

[4] Y. Gong, T. Zhao, Y. J. Rao, and Y. Wu, "All-fiber curvature sensor based on multimode interference," IEEE Photonics Technology Letters, 2011, 23(11): 679-681.

[5] A. M. Huatta, Y. L. Semenova, and G. Farrell, "Strain sensor based on a pair of single-mode-multimode-single-mode fiber structures in a ratiometric power measurement scheme," Applied Optics, 2010, 49(3): 536-541.

[6] Q. Wu, Y. L. Semenova, P. F. Wang, A. M. Hatta, and G. Farrell, "Experimental demonstration of a simple displacement sensor based on a bent single-mode-multimode-single-mode fiber structure," Measurement Science and Technology, 2011, 22(2): 025203-1-025203-5.

[7] L. Yang, L. L. Xue, D. Che, and J. G. Qian, "Guided-mode-leaky-mode-guided-mode fiber structure and its application to high refractive index sensing," Optics Letters, 2012, 37(4): 587-589.

[8] R. X. Zhang, T. G. Liu, Q. Han, Y. F. Chen, and L. Li, "U-bent single-mode-multimode-single-mode fiber optic magnetic field sensor based on magnetic fluid," Applied Physics Express, 2014, 7(7):
072501-1-072501-4.

[9] Y. Liu and L. Wei, "Low-cost high-sensitivity strain and temperature sensing using graded-index multimode fibers," Applied Optics, 2007, 46(13): 2516-2519.

[10] Q. Wu, Y. Semenova, A. M. Hatter, P. Wang, and G. Farrell, "Bent SMS fibre structure for temperature measurement," Electronics Letters, 2010, 46(16): 1129-1130.

[11] Y. J. Zhang, X. J. Tian, L. L. Xue, Q. J. Zhang, L. Yang, and B. Zhu, "Super-high sensitivity of fiber temperature sensor based on leak-mode bent SMS structure," IEEE Photonics Technology Letters, 2013, 25(6): 560-563.

[12] K. S. Lim, I. Aryanfar, W. Y. Chong, Y. K. Cheng, S. W. Harun, and H. Ahamd, "Integrated microfibre device for refractive index and temperature sensing," Sensors, 2012, 12(9): 11782-11789.

[13] J. Shi, Y. Y. Wang, D. G. Xu, H. W. Zhang, G. H. Su, L. C. Duan, et al., "Temperature sensor based on fiber ring laser with Saganc loop," IEEE Photonics Technology Letters, 2016, 28(7): 794-797.

[14] E. R. Vera, C. M. B. Cordeiro, and P. Tottes, "High sensitive temperature sensor using Saganc loop interferometer based on side-hole photonic crystal fiber filled with metal," Applied Optics, 2017, 56(2): 156-162.

[15] J. Ruan, P. Huang, Z. X. Qin, and Q. Zeng, "Temperature characteristic of SMPMS fiber structure-based Saganc loop," IEEE Photonics Technology Letters, 2014, 27(1): 62-64.

[16] H. P. Gong, C. C. Chan, L. H. Chen, and X. Y. Dong, "Strain sensor realized by using low-birefringence photonic-crystal-fiber-based Saganc loop," IEEE Photonics Technology Letters, 2010, 22(16): 1238-1240.

[17] S. Rashleigh, "Origins and control of polarization effects in single-mode fibers," Journal of Lightwave Technology, 1983, 1(2): 312-331.

[18] A. N. Starodumov, L. A. Zenteno, D. Monzon, and E. D. L. Rosa, "Fiber Saganc interferometer temperature sensor," Applied Physics Letters, 1997, 70(1): 19-21. 